\title{
DETERMINAÇÃO DA ATIVIDADE ANTIOXIDANTE DAS MATRIZES VEGETAIS Capsicum chinense, Capsicum frutescens e Tripodanthus acutifolius
}

\author{
ORTOLAN, S. A. ${ }^{\text {; }}$ HERMES, V. C. 2; PEDROSO, P. S. 2; LEAL, V. L. 3; TISCHER, B. 4 ; \\ POSSUELO, L. G.5; SILVA, C. M. ${ }^{6}$
}

PALAVRAS CHAVE: Antioxidante, ORAC, Capsicum chinense, Capsicum frutescens, Tripodanthus acutifolius.

\begin{abstract}
RESUMO
Radicais livres podem gerar estresse oxidativo e causar danos celulares. 0 uso de antioxidantes naturais demonstram ser um importante mecanismo para retardar ou inibir esse processo de oxidação. Matrizes vegetais dos gêneros Capsicum e Tripodanthus apresentam metabólitos secundários com atividade antioxidante relatada. Foi avaliada a atividade antioxidante de oleoresinas das pimentas Capsicum chinense e Capsicum frutescens, e do extrato bruto da espécie Tripodanthus acutifolius, através do ensaio Oxygen Radical Absorbance Capacity (ORAC). As oleoresinas de $C$. chinense e $C$. frutescens apresentaram valores de 550,210 $\mu \mathrm{mol} \mathrm{TE} / \mathrm{g}$ amostra e $603,746 \mu \mathrm{mol} \mathrm{TE} / \mathrm{g}$ amostra, respectivamente. A espécie $T$. acutifolius apresentou valores aproximadamente dez vezes superiores $(5277,764 \mu \mathrm{mol} \mathrm{TE} / \mathrm{g}$ amostra) às oleoresinas. Observou-se uma relação direta da presença de compostos fenólicos com a atividade antioxidante. 0 ensaio realizado pelo grupo de pesquisas gerou um procedimento operacional interno que pode ser seguido em novas pesquisas de interesse com matrizes vegetais, aprovado para utilização no Parque Científico e Tecnológico Regional da Universidade de Santa Cruz do Sul (TecnoUnisc).
\end{abstract}

\section{DETERMINATION OF THE ANTIOXIDANT ACTIVITY OF VEGETABLE MATRICES Capsicum chinense, Capsicum frutescens AND Tripodanthus acutifolius}

KEYWORDS: Antioxidant, ORAC, Capsicum chinense, Capsicum frutescens, Tripodanthus acutifolius.

\begin{abstract}
Free radicals can generate oxidative stress and cause cell damage. The use of natural antioxidants evidences to be an important mechanism to delay or inhibit this oxidation process. Vegetable matrices of the genus Capsicum and Tripodanthus have secondary metabolites with reported antioxidant activity. The antioxidant activity of Capsicum chinense and Capsicum frutescens oleoresins peppers and of the crude extract of Tripodanthus acutifolius was evaluated by the Oxygen Radical Absorbance Capacity (ORAC) assay. The samples $C$. chinense and C. frutescens oleoresins presented values of $550,210 \mu \mathrm{mol} \mathrm{TE} / \mathrm{g}$ and $603,746 \mu \mathrm{mol} \mathrm{TE} / \mathrm{g}$, respectively. The sample $T$. acutifolius presented values approximately ten times higher $(5.277,764 \mu \mathrm{mol} T E / \mathrm{g})$ to the oleoresins. It was observed a direct relation of the presence of phenolic compounds with the antioxidant activity. The research conducted by this group generated an internal operating procedure that can be adopt in new researches of interest with plant matrices. This document was approved for use in the Regional Scientific and Technological Park of the University of Santa Cruz do Sul (TecnoUnisc).
\end{abstract}

\footnotetext{
${ }_{1}^{1}$ Graduado em Farmácia pela Universidade de Santa Cruz do Sul.

2 Acadêmica do curso de Farmácia da Universidade de Santa Cruz do Sul

${ }^{3}$ Mestre em Microbiologia Agrícola e do Ambiente pela Universidade Federal do Rio Grande do Sul.

${ }^{4}$ Doutora em Ciência e Tecnologia de Alimentos pela Universidade Federal de Santa Maria.

5 Doutora em Ciências Biológicas (Bioquímica) pela Universidade Federal do Rio Grande do Sul.

${ }^{5}$ Doutora em Ciências Farmacêuticas pela Universidade Federal do Rio Grande do Sul.
} 


\section{INTRODUÇÃO}

A geração de radicais livres nos organismos vivos pode gerar estresse oxidativo, causando problemas de saúde como danos ao material genético e, consequentemente, contribuindo no surgimento de doenças hepáticas, doenças neurológicas degenerativas e até mesmo metastase cancerígena (DAIRAM et al., 2008; MANJUNATHA e SRINIVASAN, 2006). Afim de combater esses danos, estimula-se o uso de substâncias antioxidantes, uma vez que estas demonstram ser um importante mecanismo para retardar ou até mesmo inibir os processos de oxidação a nível celular (PANYA et al., 2015; ROJAS e BUITRAGO, 2019).

Matrizes vegetais são importantes fontes para obtenção de moléculas bioativas, consideradas como potentes antioxidantes naturais. Entre elas, destacam-se os compostos fenólicos (compostos fenólicos simples, ácidos fenólicos, cumarinas, flavonoides, estilbenos, taninos, lignanas, tocoferóis), compostos nitrogenados (alcaloides, aminoácidos e aminas), carotenoides e o ácido ascórbico (ROJAS e BUITRAGO, 2019).

As pimentas e pimentões do gênero Capsicum são conhecidas fontes de antioxidantes naturais, pois são ricas em compostos fenólicos denominados capsaicinóides, que são responsáveis também pelo sabor picante do fruto (DIAZ et al., 2004). A capsaicina, que é o capsaicinoide mais abundante, inibe a oxidação de lipídios de baixa densidade (LDL) induzida por ferro e cobre, baixando o nível de oxidação de biomoléculas (DAIRAM et al., 2008; MANJUNATHA e SRINIVASAN, 2006). Esse metabólito apresenta atividades antioxidante (AMMA et al., 2019; COSTA et al., 2019; LAVORGNA et al., 2019), analgésica (GERNER et al., 2008) e termogênica (JO0, 2010) comprovadas.

Tripodanthus acutifolius (Ruiz \& Pavón) Van Tieghem é uma planta hemiparasita, conhecida popularmente como erva-de-passarinho. Ela possui atividade antioxidante, antimicrobiana, anti-inflamatória, analgésica, antipirética e diurética, sendo estudas com base em seus usos na medicina popular (INTERSIMONE et al., 2005; DAUD et al., 2005; DAUD et al., 2006; SOBERÓN et al., 2007; SOBERÓN et al., 2010a; SOBERÓN et al., 2010b; SOBERÓN et al., 2010c; SILVA, 2014; SOUZA et al., 2014). Assim como para demais plantas, essas propriedades farmacológicas podem estar associadas aos metabólitos secundários de T. acutifolius, que incluem os compostos fenólicos flavonoides, taninos condensados e catequinas, além de um raro composto isolado e identificado como tripodantosídeo (SOBERÓN et al., 2010c; SILVA, 2014; COELHO et al., 2018).

A investigação fitoquímica e biológica, através da determinação e mensuração do potencial antioxidante dessas e outras substâncias, vem ganhando a atenção dos pesquisadores, uma vez que possibilita a descoberta de novas moléculas bioativas e a criação de estratégias para a síntese de novos medicamentos (ELISABETSKY; SOUZA, 2004). Ao longo dos anos, vários métodos foram propostos e testados na investigação da capacidade antioxidante das plantas (ROJAS e BUITRAGO, 2019), sendo o ensaio Oxygen Radical Absorbance Capacity (ORAC) um dos métodos mais recentes para verificação e medição dessa capacidade. Este método tem sido muito utilizado, pois acontece em condições de temperatura e pH similares as fisiológicas, diferente dos métodos com o radical DPPH e o Ferric Reducing Antioxidant Power (FRAP), que devem ser realizados em temperaturas elevadas ou pH ácidos (PANYA et al. 2015). O ORAC é um método preciso e com resultados confiáveis, sendo amplamente utilizado em pesquisas com extratos de plantas brutos ou parcialmente purificados (PHONSATTA et al., 2019).

Neste contexto, o objetivo deste trabalho foi determinar a atividade antioxidante de duas matrizes vegetais do gênero Capsicum e uma matriz vegetal da espécie $T$. acutifolius, utilizando o ensaio ORAC, assim como elaborar e disponibilizar um protocolo interno simplificado, desenvolvido com base na metodologia de Ou e 
colaboradores (2001), para a realização do ensaio ORAC com outras matrizes vegetais no laboratório de Oleoquímica do Parque Científico e Tecnológico Regional da Unisc (TecnoUnisc).

\section{MATERIAIS E MÉTODOS}

\subsection{OBTENÇÃO DAS MATRIZES VEGETAIS}

Os frutos das pimentas Habanero (Capsicum chinense Jacq., Solanaceae) e Malagueta (Capsicum frutescens L., Solanaceae) foram adquiridos no comércio regional, higienizados (100 ppm cloro livre) e secos em estufa de ar circulante (Tecnal TE-394/5) a $65^{\circ} \mathrm{C} \pm 2^{\circ} \mathrm{C}$ por 24 horas. 0 material vegetal seco foi moído em moinho de facas tipo Willye (Tecnal TE-680) e armazenado em frasco âmbar. 0 acesso ao patrimônio genético foi informado junto ao Sistema Nacional de Gestão do Patrimônio Genético e do Conhecimento Tradicional Associado (SisGen), sob cadastro $n^{\circ}$ A6A804C. A partir do material vegetal seco e moído, foram preparadas oleoresinas ricas em compostos fenólicos, através de extração por Soxhlet (AOAC 995.03), utilizando o solvente metanol, que posteriormente foi rotaevaporado (Tecnal TE-210) $\left(40^{\circ} \mathrm{C}, 50 \mathrm{rpm},-600 \mathrm{mmHg}\right)$.

As folhas da erva-de-passarinho (Tripodanthus acutifolius (Ruiz \& Pavón) Van Tieghem (Loranthaceae) foram coletadas no município de Santa Cruz do Sul, RS, identificadas e depositadas no herbário do Instituto de Biociências da Universidade Federal do Rio Grande Sul (UFRGS), sob o número ICN 167796. As folhas foram higienizadas e secas em estufa de ar circulante (Tecnal TE-394/5) a $40^{\circ} \mathrm{C}$ por 48 horas. Em seguida, foram moídas em moinho de facas tipo Willye (Tecnal TE-680) e armazenadas em frasco âmbar até a preparação do extrato. 0 acesso ao patrimônio genético foi igualmente informado junto ao SisGen, sob o cadastro $n^{\circ}$ AAFC1F0. A obtenção do extrato foi realizada através da técnica de Soxhlet, utilizando metanol como solvente, posteriormente rotaevaporado (Tecnal TE-210) $\left(40^{\circ} \mathrm{C}, 50 \mathrm{rpm},-600 \mathrm{mmHg}\right)$, obtendo-se o extrato metanólico bruto (EMB).

\subsection{ENSAIO ORAC}

O método ORAC (OU et al., 2001) avaliou a capacidade de desativação de radicais livres induzidos por 2,2'-azobis-(2-metilpropanoamidina) (AAPH) pelas espécies vegetais com capacidade antioxidante. Foram preparados o diluente tampão fosfato $75 \mathrm{mM}(\mathrm{pH} 7,4)$, a partir de fosfato de potássio dibásico e monobásico; solução estoque de fluoresceína $407 \mu \mathrm{M}$ e a solução estoque de Trolox $4000 \mu \mathrm{M}$. Posteriormente, foi realizada uma diluição seriada da solução de trolox, obtendo-se as concentrações de 2 a $96 \mu$ M (P1 a P10). As oleoresinas das pimentas e o extrato metanólico bruto de erva-de-passarinho foram solubilizados em metanol, obtendo-se uma solução inicial de $5.000 \mathrm{mg} \mathrm{L}^{-1}$. Após isso, foram diluídas no mesmo tampão fosfato, obtendo-se soluções de $50 \mathrm{mg} \mathrm{L}^{-1}$ e $20 \mathrm{mg} \mathrm{L}^{-1}$, respectivamente.

Em uma placa preta de 96 poços (Fluotrac 200, Greiner bio-one ${ }^{\circledR}$ ), foram adicionados $25 \mu \mathrm{L}$ das matrizes vegetais ou $25 \mu \mathrm{L}$ do padrão Trolox em diferentes concentrações (P1 a P10) (Figura 1). Posteriormente, foi adicionado $150 \mu \mathrm{L}$ de fluoresceína (81 nM) e o sistema foi incubado (SpectraMax® M3) por 10 minutos a 37 ${ }^{\circ} \mathrm{C}$, sendo os 3 últimos minutos sob constante agitação. Após essa etapa, foram adicionados $25 \mu \mathrm{L}$ da solução de AAPH (152 mM) como fonte geradora de radicais peroxila. Foram realizados brancos para analisar a integridade da placa, dos reagentes e ação natural do AAPH na sonda fluoresceína. 0 volume final de cada poço foi de 200 $\mu \mathrm{L}$. A fluorescência foi monitorada a cada minuto ( $\lambda$ excitação $=485 \mathrm{~nm}$ e $\lambda$ emissão $=528 \mathrm{~nm}$ ), durante 90 
minutos a $37^{\circ} \mathrm{C}$ na leitora de placas (SpectraMax ${ }^{\circ}$ M3). A capacidade antioxidante foi determinada pela área sob a curva (AUC) e os resultados foram comparados com a curva padrão de Trolox (0 - $96 \mu \mathrm{M})$ e expressos em " $\mu \mathrm{mol}$ de Trolox Equivalente (TE) por grama de amostra" e "grama de trolox por grama de amostra".

Figura 1. Esquema da microplaca para o ensaio ORAC

\begin{tabular}{|c|c|c|c|c|c|c|c|c|c|c|c|c|}
\hline & $\mathbf{1}$ & $\mathbf{2}$ & $\mathbf{3}$ & $\mathbf{4}$ & $\mathbf{5}$ & $\mathbf{6}$ & $\mathbf{7}$ & $\mathbf{8}$ & $\mathbf{9}$ & $\mathbf{1 0}$ & $\mathbf{1 1}$ & $\mathbf{1 2}$ \\
\hline A & BP & BP & BF & BF & BR & BR & BT & BT & BT & P1 & P1 & P1 \\
\hline B & P2 & P2 & P2 & P3 & P3 & P3 & P4 & P4 & P4 & P5 & P5 & P5 \\
\hline C & P6 & P6 & P6 & P7 & P7 & P7 & P8 & P8 & P8 & P9 & P9 & P9 \\
\hline D & P10 & P10 & P10 & A1 & A1 & A1 & A2 & A2 & A2 & A3 & A3 & A3 \\
\hline E & $\ldots$ & $\ldots$ & $\ldots$ & & & & & & & & & \\
\hline F & & & & & & & & & & & & \\
\hline G & & & & & & & & & & & & \\
\hline H & & & & & & & & & & & & \\
\hline
\end{tabular}

Legenda: $\mathrm{T}=$ tampão; $\mathrm{FL}=$ fluoresceína; $\mathrm{BP}=$ branco da placa $(200 \mu \mathrm{L} \mathrm{T}) ; \mathrm{BF}=$ branco da fluoresceína $(50 \mu \mathrm{L} T$ + $150 \mu \mathrm{L} F \mathrm{~F}) ; \mathrm{BR}=$ branco do radical $(175 \mu \mathrm{L} \mathrm{T}+25 \mu \mathrm{L}$ AAPH $) ; \mathrm{BT}=$ branco do tampão $(25 \mu \mathrm{L} \mathrm{T}+150 \mu \mathrm{L} \mathrm{FL}+$ $25 \mu \mathrm{L}$ AAPH); $\mathrm{P}=$ padrão $(25 \mu \mathrm{L} \mathrm{P}+150 \mu \mathrm{L} \mathrm{FL}+25 \mu \mathrm{L}$ AAPH); A= amostra $(25 \mu \mathrm{L}$ A + $150 \mu \mathrm{L} F+25 \mu \mathrm{L}$ AAPH).

\subsection{ANÁLISES ESTATÍSTICAS}

As análises foram realizadas em triplicata e os resultados obtidos foram expressos como média e desvio padrão. A análise estatística de variância (ANOVA) com post-hoc de Tukey foi realizada no programa Origin Pro 8.5 ao nível de $5 \%$ de probabilidade.

\section{RESULTADOS E DISCUSSÕES}

A fluorescência relativa medida ao longo do tempo definiu o potencial antioxidante dos compostos em relação ao padrão Trolox. As matrizes vegetais analisadas apresentaram um comportamento de queda entre os pontos da curva analítica (Figura 2), sendo medidos pela equação da reta oriunda da área sob a curva (Tabela 1).

Figura 2. Fluorescência relativa dos compostos analisados

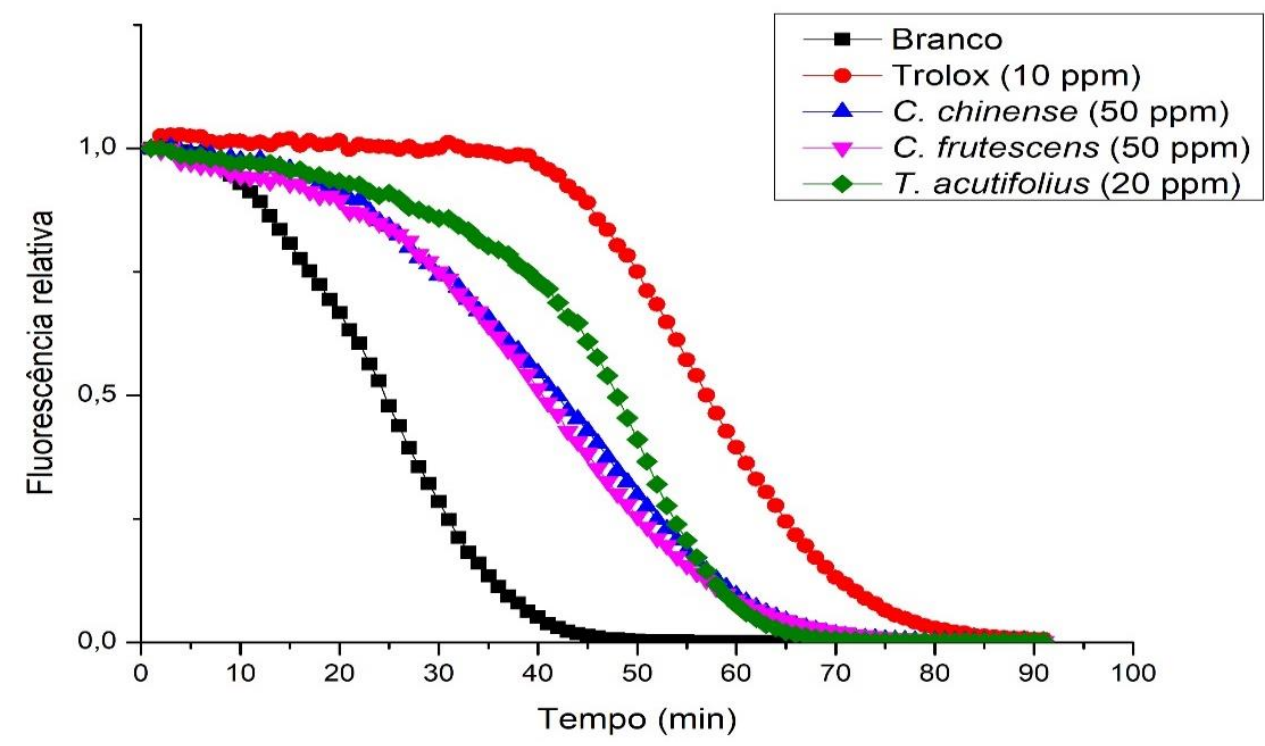


Tabela 1. Capacidade antioxidante das matrizes vegetais

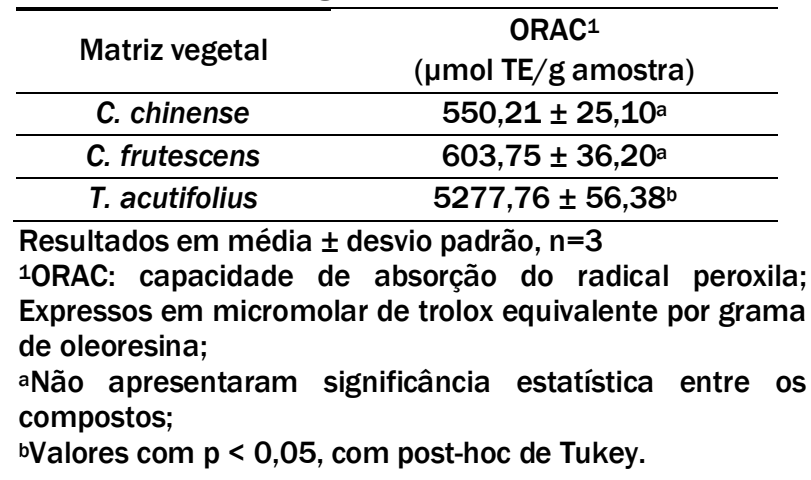

Foi observado que as matrizes vegetais analisadas apresentaram atividade antioxidante próxima ao padrão Trolox. Entretanto, o extrato vegetal obtido da espécie $T$. acutifolius $(5277,764 \mu \mathrm{mol}$ TE/g amostra) apresentou resultado aproximadamente dez vezes superior às oleoresinas de $C$. chinense $(550,210 \mu \mathrm{mol} T E / g$ amostra) e C. frutescens $(603,746 \mu \mathrm{mol} \mathrm{TE} / \mathrm{g}$ amostra).

Através do método DPPH, Silva e colaboradores (2014) avaliaram e confirmaram a atividade antioxidante in vitro do extrato aquoso das folhas de T. acutifolius, onde o extrato inibiu a formação e/ou a captura do radical livre DPPH em todas as concentrações testadas. Soberón e colaboradores (2010c) relataram uma significativa atividade de sequestro de radicais livres a partir de quatro flavonoides isolados de $T$. acutifolius (rutina, nicotiflorina, hiperosideo e isoquercitrina). Ainda, o tripodantosídeo (4-(3',4'dihidroxifenil)-2-R-butanol-2-0ß-d-lucopiranosideo), composto até então encontrado somente nesta espécie, mostrou um perfil de eliminação de radicais livres semelhante aos flavonoides. De modo geral, pode-se sugerir que tal atividade biológica está intimamente relacionada à composição química desta planta, principalmente aos compostos de seu metabolismo secundário, como os flavonoides, que provaram ser altamente eficazes capturadores da maioria das moléculas oxidantes, incluindo moléculas reativas de oxigênio e radicais livres (BRAVO, 1998).

A capsaicina, que é o capsaicinoide majoritário das pimentas $C$. chinense e $C$. frutescens, demonstrou ser um potente antioxidante, reduzindo a peroxidação lipídica induzida por ferro (DAIRAM et al., 2008). As oleoresinas analisadas nesse estudo apresentaram valores semelhantes às pimentas da espécie Jalapenho (Capsicum annuum) cultivadas em estufa (424 a 587 mmol TE/g amostra) (KAVGA et al., 2018).

Quando comparados ao padrão utilizado, deve-se dosar 7,26 g da oleoresina de C. chinense, 6,61 g da oleoresina de $C$. frutescens e apenas $0,75 \mathrm{~g}$ de extrato de $T$. acutifolius para o efeito antioxidante equivalente a 1 g do padrão Trolox.

Outras matrizes vegetais ricas em compostos fenólicos e mensuradas pelo ensaio ORAC, como o orégano (Origanum vulgare) e a cerejeira-de-Santa-Lúcia (Prunus mahaleb), apresentaram atividade antioxidante, com valores de $1233 \mu \mathrm{mol} \mathrm{TE} / \mathrm{g}$ amostra (SU et al., 2007) e 518,6 $\mathrm{mmol}$ de TE/g (BLANDO et al., 2015), respectivamente.

Observou-se uma relação direta da presença de compostos fenólicos com a atividade antioxidante. Os metabólitos secundários das folhas de $T$. acutifolius contribuíram de maneira significativa para o potencial dessa atividade, enquanto os compostos fenólicos dos frutos de pimenta apresentaram atividade antioxidante mais baixo, porém de acordo com outros resultados já descritos na literatura e esperados para as pimentas (KAVGA et al., 2018; KONCZAK et al., 2010). 
O ensaio ORAC, realizado pelo grupo de pesquisa gerou um procedimento operacional interno (apresentado no material suplementar), que pode ser seguido em novas pesquisas de interesse com matrizes vegetais. Esse protocolo confere maior reprodutibilidade e, consequentemente, maior segurança ao ensaio ORAC, pois apresenta informações detalhadas sobre o preparo das soluções e configurações dos equipamentos, assim como sobre cuidados no procedimento analítico e informações sobre a viabilidade dos resultados. Além disso, a planilha com fórmulas protegidas para realização do cálculo da atividade antioxidante confere agilidade na etapa pós-analítica.

\section{CONSIDERAÇÕES FINAIS}

Tendo em vista a obtenção de resultados positivos para atividade antioxidante das matrizes vegetais avaliadas, aprovou-se a utilização do método proposto e testado nos laboratórios do Parque Científico e Tecnológico Regional da Universidade de Santa Cruz do Sul. Ressalta-se que outros compostos bioativos existentes em outras matrizes vegetais também poderão ser testados.

\section{AGRADECIMENTOS}

Os autores agradecem ao CNPq e a SDECT/RS pelo apoio financeiro e a UNISC e ao Parque Científico e Tecnológico Regional da UNISC (TecnoUnisc) pela viabilização do estudo.

\section{REFERÊNCIAS}

AMMA, T.; GHARSAN, F. N.; SHANG, K.; HASSAN, M. S.; KHIL, M.; HWANG, I. Electrospun Twin Fibers Encumbered with Intrinsic Antioxidant Activity as Prospective Bandage. Macromolecular Research, p. 1-7, 2019.

BLANDO, F.; ALBANO, C.; LIU, Y.; NICOLETTI, I.; CORRADINI, D.; TOMMASI, N.; GERARDI, C.; MITA, G.; KITTS, D. D. Polyphenolic composition and antioxidant activity of the under-utilised Prunus mahaleb L. fruit. Journal of the Science of Food and Agriculture, n.96, p.2641-2649, 2015.

BRAVO, L. Polyphenols: chemistry, dietary sources, metabolism and nutritional significance. Nutrition Reviews, v.56, p. 317-333, 1998.

COELhO, R. P.; FEKSA, D. L.; OLIVEIRA, P. M.; GÜLLICH, A. A. C.; PILAR, B. C.; PICCOLI, J. C. E.; MANFREDINI, V. Protective effect of the hydroalcoholic extract of Tripodanthus acutifolius in hypercholesterolemic Wistar rats. Biomedicine \& Pharmacotherapy, n.97, p.300-309, 2018.

COSTA, L. M.; MOURA, N. F.; Marangoni, C.; Mendes, C. E.; TEIXEIRA, A.O. Atividade antioxidante de pimentas do gênero Capsicum. Ciência e Tecnologia de Alimentos, 30(1). 2010.

DAUD, A.; GALLO, A.; RIERA, A.S. Antimicrobial properties of Phrygilanthus acutifolius. Journal of Ethnopharmacology, v.99, p. 193-197. 2005.

DAUD, A.; HABIB, N.; RIERA, A.S. Anti-inflammatory, anti-nociceptive and antipyretic effects of extracts of Phrygilanthus acutifolius flowers. Journal of Ethnopharmacology, v. 108, p. 198-203, 2006

DAIRAM, A.; FOGEL, R. DAYA, S.; LIMSON, J L. Antioxidant and iron-binding properties of curcumin, capsaicin, and s-allylcysteine reduce oxidative stress in rat brain homogenate. Journal of Agricultural and Food Chemistry, v. 56, p.3350-3356, 2008.

DÍAZ, J.; POMAR, F.; BERNAL, A.; MERINO, F. Peroxidases and the metabolism of capsaicin in Capsicum annuum. Phytochemistry Reviews, v.3, n.1-2, p.141-157, 2004. 
ELISABETSKY, E.; SOUZA, G. C. Etnofarmacologia como ferramenta na busca de substâncias ativas. In: SIMÕES. M. O.; SCHENKEL, E. P.; GOSMANN, G.; MELLO, J. C. P.; MENTZ, L. A.; PETROVICK, P. R. (Org.). Farmacognosia: da planta ao medicamento. 5. ed. Porto Alegre/Florianópolis: Editora da UFRGS / Editora da UFSC. p.107-122, 2004.

GERNER, p.; BINSHTOK, A. M.; WANG, C.; HEVELONW, N. D.; PEAN, B. P.; WOOLF, C. J.; WANG, G. K. Capsaicin combined with local anesthetics preferentially prolongs sensory/nociceptive block in rat sciatic nerve. Anesthesiology, n.109, p.872-878, 2008.

INTERSIMONE, N.H.; THOENE, A.D.; RIERA, A.S. Efecto diurético de extractos acuosos y alcohólicos de flores de Phrygilanthus acutifolius (corpo) en ratas. Revista Cubana Plantas Medicinales, v.10, p. 3-4. 2005.

JOO, J. I; KIM, D. H.; CHOL, J..; YUN, J. W. Proteomic analysis for antiobesity potential of capsaicin on white adipose tissue in rats fed with a high fat diet. Journal of Proteome Research, v.9, p.2977-2987, 2010.

KAVGA, A.; STRATI, I. F.; SINANOGLOU, V. J.; FOTAKIS, C.; SOTIROUDIS, G.; CHRISTODOULOU, P.; ZOUMPOULAKIS, $P$. Evaluating the experimental cultivation of peppers in low-energy-demand greenhouses. An interdisciplinary study. Journal of the Science of Food and Agriculture, n. 99, p.781-789, 2018.

KONCZAK, I.; ZABARAS, D.; DUNSTAN, M.; AGUAS, P. Antioxidant capacity and phenolic compounds in commercially grown native Australian herbs and spices. Food Chemistry, v.122, p.260-266, 2010.

LAVORGNA, M.; ORLO, E.; NUGNES, R.; PISCITELLI, C.; RUSSO, C.; ISIDORI, M. Capsaicin in Hot Chili Peppers: In Vitro Evaluation of Its Antiradical, Antiproliferative and Apoptotic Activities. Plant Foods for Human Nutrition, p. 17, 2019.

MANJUNATHA, H.; SRINIVASAN, K. Protective effect of dietary curcumin and capsaicin on induced oxidation of low-density lipoprotein, iron-induced hepato-toxicity and carrageenan-induced inflammation in experimental rats. The FEBS Journal., v.273, p.4528-4537, 2006.

OU, B.; HAMPSCH-WOODILL, M.; PRIOR, R. L. Development and Validation of an Improved Oxygen Radical Absorbance Capacity Assay Using Fluorescein as the Fluorescent Probe. Journal of Agricultural and Food Chemistry, v.49, p.4619-4626, 2001.

PANYA, A; TEMTHAWEe, W.; PHONSATtA, N.; CHAROENSUK, D.; DEETAE, P.; VISESSANGUAN, W.; DECKER, E. Apolar Radical Initiated Conjugated Autoxidizable Triene (ApoCAT) Assay: Effects of Oxidant Locations on Antioxidant Capacities and Interactions. Journal of agricultural and food chemistry, v. 76, p.7546-7555, 2015.

PHONSATTA, N.; LUANGPITUKSA, P.; FIGUEROA-ESPINOZA, M.C.; LECOMTE, J.; DURAND, E.; VILLENEUVE, P.; VISESSANGUAN, W.; DEEATAE, P.; UAWISETWATHANA, U.; PONGPRAYOON, W.; PANYA, A. Conjugated Autoxidizable Triene-Based (CAT and ApoCAT) Assays: Their Practical Application for Screening of Crude Plant Extracts with Antioxidant Functions in Relevant to Oil-in-Water Emulsions. European Journal of lipid science and technology, v. 121, p.1-11, 2019.

ROJAS, Janne; BUITRAGO, Alexis. Antioxidant Activity of Phenolic Compounds Biosynthesized by Plants and Its Relationship With Prevention of Neurodegenerative Diseases. In: Bioactive Compounds. Woodhead Publishing, p. 3-31. 2019.

SILVA, C. M. Estudo químico e biológico de Tripodanthus acutifolius (Ruiz \& Pav.) Tiegh., Loranthaceae. Tese (Programa de Pós-graduação em Ciências Farmacêuticas) Universidade Federal do Rio Grande do Sul. Porto Alegre. 2014.

SILVA, M. L. C; COSTA, R. S.; SANTANA, A. S.; KOBLITZ, M. G. B. Compostos fenólicos, carotenóides e atividade antioxidante em produtos vegetais. Semina: Ciências Agrárias, v. 31, n.3, p. 669-682, 2010.

SOBERÓN, J.R.; SGARIGLIA, M.A.; SAMPIETRO, D.A.; QUIROGA, E.N.; VATTUONE, M.A. Antibacterial activity of plant extracts from northwestern Argentina. Journal of Applied Microbiology, v. 102, p. 1450-1461. 2007.

SOBERÓN, J.R.; SGARIGLIA, M.A.; SAMPIETRO, D.A.; QUIROGA, E.N.; VATTUONE, M.A. Study of antiinflammatory activity of metabolites isolated from Tripodanthus acutifolius. Molecular Medicinal Chemistry, v. 21, p. 88-90, 2010a. 
SOBERÓN, J.R.; SGARIGLIA, M.A.; SAMPIETRO, D.A.; QUIROGA, E.N.; VATTUONE, M.A. Free radical scavenging activities and inhibition of inflammatory enzymes of phenolics isolated from Tripodanthus acutifolius. Journal of Ethnopharmacology, v. 130, p. 329- 333, 2010 b.

SOBERÓN, J.R.; SGARIGLIA, M.A.; SAMPIETRO, D.A.; QUIROGA, E.N.; SIERRA, M.G.; VATTUONE, M.A. Purification and identification of antibacterial phenolics from Tripodanthus acutifolius leaves. Journal of Applied Microbiology, v.108, p. 1757-1768, 2010c.

SOUZA, T. K.; SILVA, C. M.; BENITEZ, L. B.; VON POSER, G. L.; ZUANAZZI, J. A. S. Atividade antibacteriana do extrato aquoso de Tripodanthus acutifolius frente a Staphylococcus aureus. Revista Jovens Pesquisadores, v. 4, n. 1, p. 618, 2014.

SU, L.; YIN, J.; CHARLES, D.; ZHOU, K.; MOORE, J. YU, L.; Total phenolic contents, chelating capacities, and radicalscavenging properties of black peppercorn, nutmeg, rosehip, cinnamon and oregano leaf. Food Chemistry, v.100, p.990-997, 2007. 